\title{
NullaNet Tiny: Ultra-low-latency DNN Inference Through Fixed-function Combinational Logic
}

\author{
Mahdi Nazemi*, Arash Fayyazi*, Amirhossein Esmaili, \\ Atharva Khare, Soheil Nazar Shahsavani, and Massoud Pedram \\ School of Electrical and Computer Engineering \\ University of Southern California, Los Angeles, California 90089-0001 \\ Email: \{mnazemi, fayyazi, esmailid, aakhare, nazarsha, pedram\}@usc.edu
}

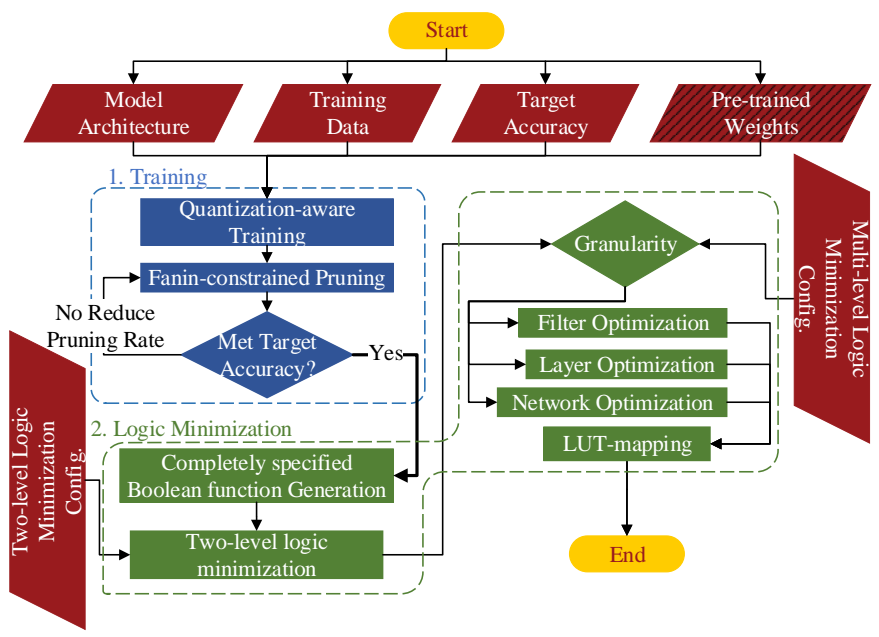

Fig. 1. A high-level overview of NullaNet Tiny's design and optimization flow.

\section{EXTENDED ABSTRACT}

While there is a large body of research on efficient processing of deep neural networks (DNNs) [1]-[31], ultra-low-latency realization of these models for applications with stringent, sub-microsecond latency requirements continues to be an unresolved, challenging problem. Field-programmable gate array (FPGA)-based DNN accelerators are gaining traction as a serious contender to replace graphics processing unit/central processing unit-based platforms considering their performance, flexibility, and energy efficiency. NullaNet (2018) [32], LUTNet (2019) [33], and LogicNets (2020) [34] are among accelerators specifically designed to benefit from FPGAs' capabilities.

This paper presents NullaNet Tiny, an across-the-stack design and optimization framework for constructing resource and energyefficient, ultra-low-latency FPGA-based neural network accelerators. The key idea is to replace expensive operations required to compute various filter/neuron functions in a DNN with Boolean logic expressions that are mapped to the native look-up tables (LUTs) of the FPGA device (examples of such operations are multiply-andaccumulate and batch normalization).

Fig. 1 depicts different steps of NullaNet Tiny's design and optimization flow. The training module is responsible for both quantization-aware training (QAT) and fanin-constrained pruning (FCP) while the logic minimization module performs two-level and multi-level logic minimization in addition to retiming. These modules' ultimate goal is to convert each filter/neuron into a highly

*M. Nazemi and A. Fayyazi contributed equally to this work.
TABLE I

COMPARISON OF ACCURACY \& HARDWARE REALIZATION METRICS OF NullaNet Tiny AND LOGICNETS [34] ON THE JSC TASK.

\begin{tabular}{|c|c|c|c|c|}
\hline Arch. & $\begin{array}{c}\text { Accuracy } \\
\text { (\% Inc.) }\end{array}$ & $\begin{array}{l}\text { LUTs } \\
\text { (Dec.) }\end{array}$ & $\begin{array}{c}\text { FFs } \\
\text { (Dec.) }\end{array}$ & $\begin{array}{c}f_{\max } \\
\text { (Inc.) }\end{array}$ \\
\hline JSC-S & $\begin{array}{l}69.65 \% \\
(+\mathbf{1 . 8 5})\end{array}$ & $\begin{array}{c}39 \\
(\mathbf{5 . 5 0} \times)\end{array}$ & $\begin{array}{c}75 \\
(3.30 \times)\end{array}$ & $\begin{array}{c}2,079 \mathrm{MHz} \\
(\mathbf{1 . 3 0} \times)\end{array}$ \\
\hline JSC-M & $\begin{array}{l}72.22 \% \\
(+\mathbf{1 . 7 3})\end{array}$ & $\begin{array}{c}1,553 \\
(\mathbf{9 . 3 0} \times)\end{array}$ & $\begin{array}{c}151 \\
(\mathbf{2 . 9 0} \times)\end{array}$ & $\begin{array}{l}841 \mathrm{MHz} \\
(\mathbf{1 . 4 0 \times})\end{array}$ \\
\hline JSC-L & $\begin{array}{l}73.35 \% \\
(+\mathbf{1 . 5 5})\end{array}$ & $\begin{array}{c}11,752 \\
(3.20 \times)\end{array}$ & $\begin{array}{c}565 \\
(1.40 \times)\end{array}$ & $\begin{array}{l}436 \mathrm{MHz} \\
(\mathbf{1 . 0 2} \times)\end{array}$ \\
\hline
\end{tabular}

optimized LUT-based implementation by enumerating all its possible input combinations, recording encountered output values, and optimizing truth tables reflecting those input-output combinations.

QAT refers to the quantization of activations to binary, bipolar, or multi-bit values during neural network training. One of the critical differences between NullaNet Tiny and prior work is that it employs different activation functions for different layers to yield higher accuracy. For example, if the inputs to a DNN assume both negative and positive values, it uses an activation function such as the sign function to capture the range of inputs better. On the other hand, if a set of values can only assume non-negative numbers, it relies on the parameterized clipping activation (PACT) [9] function to quantize activations.

FCP applies fanin constraints to individual filters/neurons such that the number of inputs to each filter/neuron is small enough to make a realization based on input enumeration (as described in NullaNet [32]) feasible. In this work, FCP is either based on the alternating direction method of multipliers [35] or gradual pruning [11].

Finally, functions of different filters/neurons are represented using truth tables which are then fed to the logic minimization module. This paper employs the ESPRESSO-II logic minimizer [36] for twolevel logic minimization and Xilinx Vivado for multi-level logic minimization and retiming.

Our experimental evaluations summarized in Table I demonstrate the superior performance of NullaNet Tiny in terms of accuracy, inference latency, and resource utilization compared to prior art FPGA-based DNN accelerators on the jet substructure classification (JSC) [37] task (the three architectures are based on those described in LogicNets [34]). At about the same level of classification accuracy, compared to Xilinx's LogicNets [34], our design achieves $2.36 \times$ lower latency and $24.42 \times$ lower LUT utilization (please note that not all reported clock frequencies are realizable on the target Xilinx VU9P FPGA). Similarly, compared to Google's optimized design [38], our design has a $9.25 \times$ lower latency. 


\section{REFERENCES}

[1] V. Sze et al., "Efficient processing of deep neural networks: A tutorial and survey," Proceedings of the IEEE, vol. 105, no. 12, pp. 2295-2329, 2017. [Online]. Available: https://doi.org/10.1109/JPROC.2017.2761740

[2] M. Rastegari et al., "XNOR-Net: ImageNet classification using binary convolutional neural networks," in European Conference on Computer Vision, ser. Lecture Notes in Computer Science, vol. 9908. Springer, 2016, pp. 525-542. [Online]. Available: https://doi.org/10.1007/978-3-319-46493-0_32

[3] I. Hubara et al., "Binarized neural networks," in Advances in Neural Information Processing Systems, 2016, pp. 4107-4115. [Online]. Available: https://papers.nips.cc/paper/6573-binarized-neural-networks

[4] S. Zhou et al., "DoReFa-Net: Training low bitwidth convolutional neura networks with low bitwidth gradients," CoRR, vol. abs/1606.06160, 2016. [Online]. Available: https://arxiv.org/abs/1606.06160

[5] F. Li and B. Liu, "Ternary weight networks," CoRR, vol. abs/1605.04711, 2016. [Online]. Available: https://arxiv.org/abs/1605.04711

[6] A. Zhou et al., "Incremental network quantization: Towards lossless CNNs with low-precision weights," in International Conference on Learning Representations. OpenReview.net, 2017. [Online]. Available: https://openreview.net/forum?id=HyQJ-mclg

[7] C. Zhu et al., "Trained ternary quantization," in International Conference on Learning Representations. OpenReview.net, 2017. [Online]. Available: https://openreview.net/forum?id=S1_pAu9x1

[8] A. K. Mishra et al., "WRPN: wide reduced-precision networks," in International Conference on Learning Representations. OpenReview.net, 2018. [Online]. Available: https://openreview.net/forum?id=B1ZvaaeAZ

[9] J. Choi et al., "PACT: parameterized clipping activation for quantized neural networks," CoRR, vol. abs/1805.06085, 2018. [Online]. Available: https://arxiv.org/abs/1805.06085

[10] S. Han et al., "Learning both weights and connections for efficient neural networks," CoRR, vol. abs/1506.02626, 2015. [Online]. Available: https://arxiv.org/abs/1506.02626

[11] M. Zhu and S. Gupta, "To prune, or not to prune: Exploring the efficacy of pruning for model compression," in International Conference on Learning Representations. OpenReview.net, 2018. [Online]. Available: https://openreview.net/forum?id=SyliIDkPM

[12] T. Zhang et al., "A systematic DNN weight pruning framework using alternating direction method of multipliers," in European Conference on Computer Vision, ser. Lecture Notes in Computer Science, vol. 11212. Springer, 2018, pp. 191-207. [Online]. Available: https://doi.org/10.1007/978-3-030-01237-3_12

[13] T. Dettmers and L. Zettlemoyer, "Sparse networks from scratch: Faster training without losing performance," CoRR, vol. abs/1907.04840, 2019. [Online]. Available: https://arxiv.org/abs/1907.04840

[14] X. Ding et al., "Global sparse momentum SGD for pruning very deep neural networks," in Advances in Neural Information Processing Systems, 2019, pp. 6379-6391. [Online]. Available: https://papers.nips.cc/paper/8867-global-sparse-momentumsgd-for-pruning-very-deep-neural-networks

[15] G. E. Hinton et al., "Distilling the knowledge in a neural network," CoRR, vol. abs/1503.02531, 2015. [Online]. Available: https://arxiv.org/abs/1503.02531

[16] A. K. Mishra and D. Marr, "Apprentice: Using knowledge distillation techniques to improve low-precision network accuracy," in International Conference on Learning Representations. OpenReview.net, 2018. [Online]. Available: https://openreview.net/forum?id=B1ae11ZRb

[17] A. Polino et al., "Model compression via distillation and quantization," in International Conference on Learning Representations. OpenReview.net, 2018. [Online]. Available: https://openreview.net/forum?id=S1XolQbRW

[18] L. Theis et al., "Faster gaze prediction with dense networks and fisher pruning," CoRR, vol. abs/1801.05787, 2018. [Online]. Available: https://arxiv.org/abs/1801.05787

[19] N. Rotem et al., "Glow: Graph lowering compiler techniques for neural networks," CoRR, vol. abs/1805.00907, 2018. [Online]. Available: https://arxiv.org/abs/1805.00907

[20] T. Chen et al., "TVM: an automated end-to-end optimizing compiler for deep learning," in USENIX Symposium on Operating Systems Design and Implementation. USENIX Association, 2018, pp. 578-594. [Online]. Available: https://www.usenix.org/conference/osdi18/presentation/chen

[21] H. Sharma et al., "From high-level deep neural models to fpgas," in International Symposium on Microarchitecture. IEEE
Computer Society, 2016, pp. 17:1-17:12. [Online]. Available: https: //doi.org/10.1109/MICRO.2016.7783720

[22] S. I. Venieris and C. Bouganis, "fpgaConvNet: Mapping regular and irregular convolutional neural networks on FPGAs," IEEE Transaction on Neural Networks and Learning Systems, vol. 30, no. 2, pp. 326-342, 2019. [Online]. Available: https://doi.org/10.1109/ TNNLS.2018.2844093

[23] V. Gokhale et al., "A 240 G-ops/s mobile coprocessor for deep neural networks," in Conference on Computer Vision and Pattern Recognition. IEEE Computer Society, 2014, pp. 696-701. [Online]. Available: https://doi.org/10.1109/CVPRW.2014.106

[24] Z. Du et al., "ShiDianNao: shifting vision processing closer to the sensor," in International Symposium on Computer Architecture. ACM, 2015, pp. 92-104. [Online]. Available: https://doi.org/10.1145/ 2749469.2750389

[25] C. Zhang et al., "Optimizing FPGA-based accelerator design for deep convolutional neural networks," in International Symposium on Field-Programmable Gate Arrays. ACM, 2015, pp. 161-170. [Online]. Available: https://doi.org/10.1145/2684746.2689060

[26] Y. Chen et al., "Eyeriss: A spatial architecture for energy-efficient dataflow for convolutional neural networks," in International Symposium on Computer Architecture. IEEE Computer Society, 2016, pp. 367-379. [Online]. Available: https://doi.org/10.1109/ISCA.2016.40

[27] X. Yang et al., "DNN dataflow choice is overrated," CoRR, vol. abs/1809.04070, 2018. [Online]. Available: https://arxiv.org/abs/ 1809.04070

[28] D. Kim et al., "Neurocube: A programmable digital neuromorphic architecture with high-density 3D memory," in International Symposium on Computer Architecture. IEEE Computer Society, 2016, pp. 380-392. [Online]. Available: https://doi.org/10.1109/ISCA.2016.41

[29] M. Gao et al., "TETRIS: scalable and efficient neural network acceleration with 3D memory," in International Conference on Architectural Support for Programming Languages and Operating Systems, Y. Chen et al., Eds. ACM, 2017, pp. 751-764. [Online]. Available: https://doi.org/10.1145/3037697.3037702

[30] A. Shafiee et al., "ISAAC: A convolutional neural network accelerator with in-situ analog arithmetic in crossbars," in International Symposium on Computer Architecture. IEEE Computer Society, 2016, pp. 14-26. [Online]. Available: https://doi.org/10.1109/ISCA.2016.12

[31] P. Chi et al., "PRIME: A novel processing-in-memory architecture for neural network computation in ReRAM-based main memory," in International Symposium on Computer Architecture. IEEE Computer Society, 2016, pp. 27-39. [Online]. Available: https://doi.org/10.1109/ ISCA.2016.13

[32] M. Nazemi et al., "Energy-efficient, low-latency realization of neural networks through Boolean logic minimization," in Asia and South Pacific Design Automation Conference. ACM, 2019, pp. 274-279.

[33] E. Wang et al., "LUTNet: Rethinking inference in FPGA soft logic," in International Symposium on Field-Programmable Custom Computing Machines. IEEE, 2019, pp. 26-34. [Online]. Available: https://doi.org/10.1109/FCCM.2019.00014

[34] Y. Umuroglu et al., "LogicNets: co-designed neural networks and circuits for extreme-throughput applications," in International Conference on Field-Programmable Logic and Applications. IEEE, 2020, pp. 291297.

[35] S. P. Boyd et al., "Distributed optimization and statistical learning via the alternating direction method of multipliers," Foundations and Trends in Machine Learning, vol. 3, no. 1, pp. 1-122, 2011. [Online]. Available: https://doi.org/10.1561/2200000016

[36] R. K. Brayton et al., Logic Minimization Algorithms for VLSI Synthesis, ser. The Kluwer International Series in Engineering and Computer Science. Springer, 1984, vol. 2. [Online]. Available: https://doi.org/10.1007/978-1-4613-2821-6

[37] J. M. Duarte et al., "Fast inference of deep neural networks in FPGAs for particle physics," CoRR, vol. abs/1804.06913, 2018. [Online]. Available: https://arxiv.org/abs/1804.06913

[38] C. Coelho et al., "Automatic deep heterogeneous quantization of deep neural networks for ultra low-area, low-latency inference on the edge at particle colliders," CoRR, vol. abs/2006.10159, 2020. [Online]. Available: https://arxiv.org/abs/2006.10159 